\title{
Hábitos alimenticios en estudiantes de Nutrición de la Universidad Autónoma de Aguascalientes en el periodo enero- junio de 2015
}

Herrada-Lemus Jessica Viridiana*, Casillas-Bonilla Ana Laura*, García-Magdaleno Dora*, Ramírez-Martínez María Micaela*, Reyes-Ruiz Esparza Irma Ivonne*, Martín del Campo-Cervantes Judith**

\begin{tabular}{|c|c|}
\hline Resumen & Abstract \\
\hline 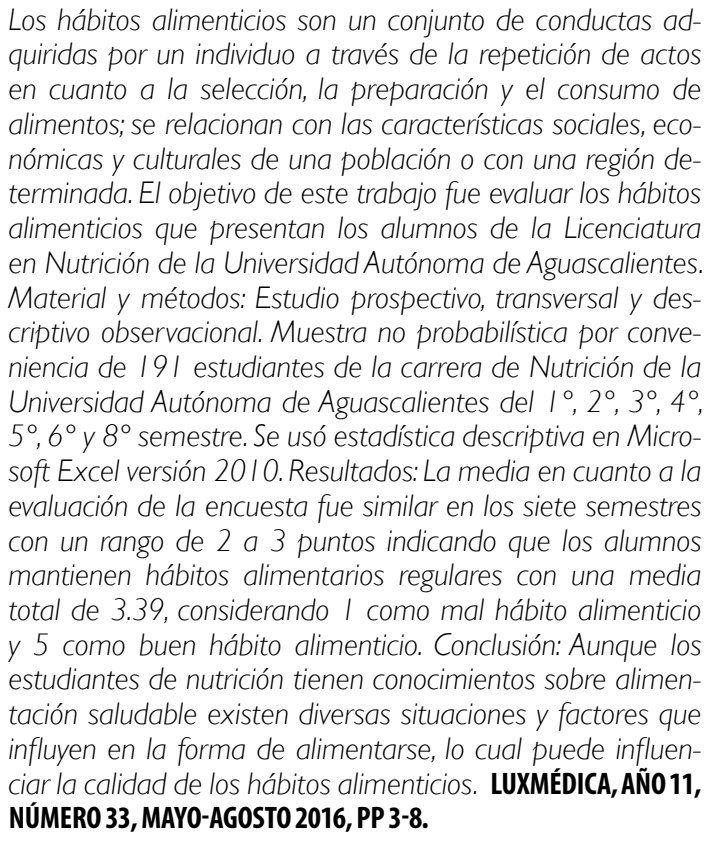 & 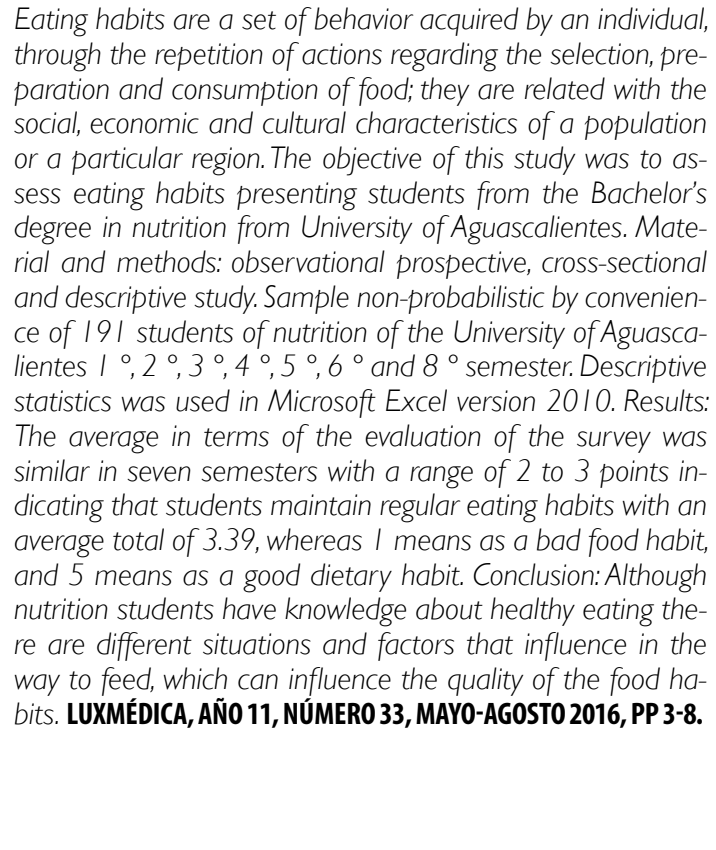 \\
\hline $\begin{array}{r}\text { Palabras clave: Hábitos alimenticios, universitarios, estu- } \\
\text { diantes de nutrición. }\end{array}$ & Keywords: food habits, University, students of nutrition. \\
\hline
\end{tabular}

* Estudiantes del séptimo semestre de la Licenciatura en Nutrición de la Universidad Autónoma de Aguascalientes.

** Maestra en Ciencias de la Nutrición, Profesora investigadora del Departamento de Nutrición y Cultura Física del Centro de Ciencias de la Salud de la Universidad Autónoma de Aguascalientes.

Fecha de recibido: 29 de septiembre 2015

Fecha de aceptación: 28 de febrero 2016

Correspondencia: MCN Judith Martín del Campo Cervantes. Edificio 107, planta baja. Campus Universitario. Avenida Universidad \#940. Código postal 20131, Aguascalientes, Ags., México. Teléfono 449 9108435. Correo electrónico jmartic@correo.uaa.mx 


\section{Introducción}

Los hábitos alimenticios son la expresión de las creencias y las tradiciones, ligados al medio geográfico y la disponibilidad alimentaria. ${ }^{1}$ El ingreso a la universidad genera una serie de cambios en la vida de los jóvenes que van desde separarse de su familia, en el caso de los estudiantes que tienen que desplazarse a las ciudades para estudiar; además de la adaptación a nuevas normas, a compañeros de estudio, a niveles elevados de estrés y a la organización del tiempo. Dentro de estos cambios, la alimentación es uno de los factores que generan mayores problemas en la salud a mediano y a largo plazo; debido a que en la etapa y ambiente universitario se reforzarán los hábitos alimenticios, ya sean buenos o malos. ${ }^{2}$ Debe destacarse también que la alimentación está determinada por ciertos factores como el nivel socioeconómico, la disponibilidad de alimentos, el omitir tiempos de comida, el poco tiempo para comer, los costos elevados, el comer entre horas, la ingesta de comida rápida y el consumo frecuente de alcohol. Todos estos conforman las costumbres alimentarias de los jóvenes universitarios que son parte importante de su conducta estudiantil. ${ }^{3}$

Montero Bravo y colaboradores dieron a conocer en su estudio que los alumnos de la Diplomatura en Nutrición Humana y Dietética tenían mayor conocimiento sobre la composición nutricional de la dieta y encontraron que esta población no sigue las recomendaciones para el consumo de los carbohidratos, las grasas y la fibra, lo que puede indicar que el tener conocimientos en nutrición no es suficiente para tener buenos hábitos alimenticios. ${ }^{1}$ El objetivo de este trabajo fue evaluar los hábitos alimenticios de los alumnos de la Licenciatura en Nutrición de la Universidad Autónoma de Aguascalientes.

\section{| | | | | | | | | | | | | | | | | | | | | | | | | | | | | | | | | | | | | | | | | | | | | | | | | | | | | | | | | | | | | | | | | | | | | | | | | | | | | | | | | | | | | | | | | | | | | | | | | | | | | | | | | |}

\section{Material y métodos}

Estudio piloto prospectivo, transversal, descriptivo y observacional. Muestreo no probabilístico por conveniencia de 191 alumnos de la carrera de Nutrición de la Universidad Autónoma de Aguascalientes. La metodología utilizada fue que los alumnos contestaran un cuestionario que está compuesto por 37 preguntas, cada pregunta tiene 5 posibilidades de respuesta, las preguntas número 1-12, 15-19, 32-34 se codifican asignándoles un valor de 1 a 5 del modo siguiente: nunca $=1$; pocas veces $=2$; alguna frecuencia $=3$; muchas veces $=4 ;$ siempre $=5$. Las preguntas 6 ,
$13,14,25-31$ se codifican en forma inversa, del siguiente modo: nunca $=5$; pocas veces $=4$; alguna frecuencia $=3$; muchas veces $=2$; siempre $=1$. Las preguntas 20-24 se valoran del siguiente modo: a la respuesta con alguna frecuencia se le da el valor 5; a las respuestas pocas veces y muchas veces se les asigna el valor 3, ya las respuestas nunca y siempre se les da un valor de 1. Por último, las preguntas 35 y 36 se valoran como sigue: nunca $=$ 5; pocas veces $=4$; alguna frecuencia $=3$; muchas veces $=2$; siempre $=1$. La puntuación total del cuestionario y de cada uno de sus factores puede obtenerse como el 
promedio de la ponderación de cada una de las preguntas incluidas, así como los resultados obtenidos serán "buenos, regulares o malos" ${ }^{4}$. En el cuestionario se evalúan ocho factores asociados a una dieta más saludable: consumo de azúcar, alimentación saludable, ejercicio físico, contenido calórico de los alimentos, bienestar psicológico, tipo de alimentos, conocimiento y control sobre lo que consume e ingesta de alcohol. ${ }^{4} \mathrm{~A}$ los resultados se les aplicó estadística descriptiva con el programa Microsoft Excel versión 2010.

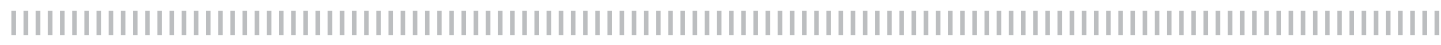

\section{Resultados}

Se estudió a 191 alumnos de la Licenciatura en Nutrición de la Universidad Autónoma de Aguascalientes, durante mayo de 2015. La distribución por semestres y género se encuentra en la figura 1 , la edad promedio fue de 20.52 años (DE \pm 1.9 años).

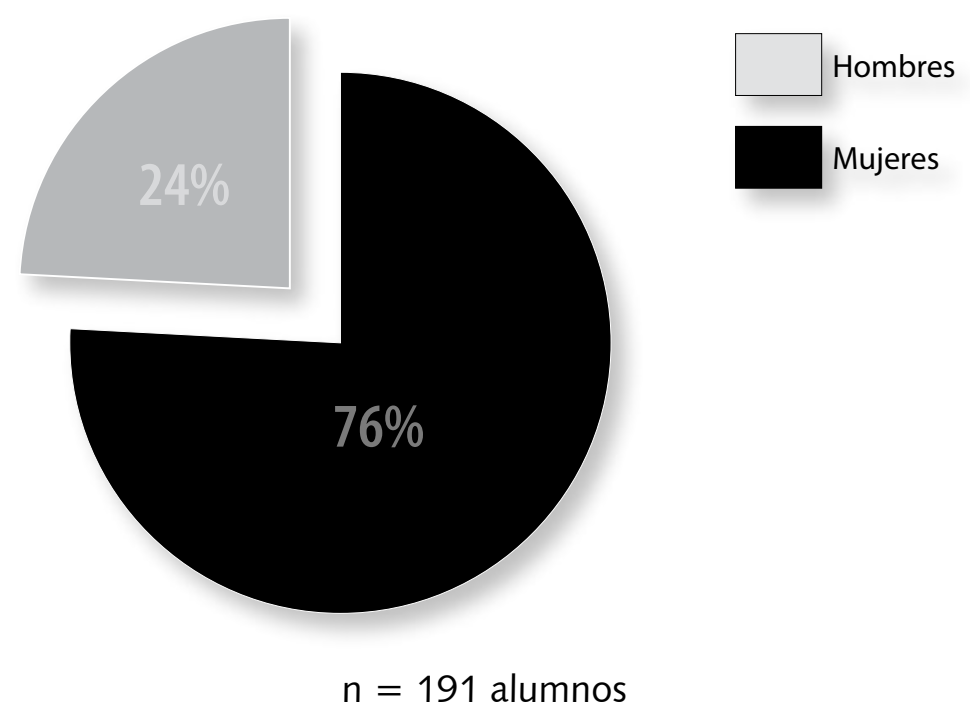

Figura 1. Distribución de la muestra, según la condición de sexo.

La encuesta evaluada tiene un puntaje del 1 al 5, indicando 1 como mal hábito alimenticio, de 2 a 3 como regular y de 4 a 5 como buen hábito alimenticio. La media en cuanto a la evaluación de la encuesta fue similar en los siete semestres estudiados con un rango de 2 a 3 puntos indicando que los alumnos mantienen hábitos alimenticios regulares con una media total de 3.39 .
En cuanto a los ocho factores valorados en la encuesta se obtuvieron medias parecidas, confirmando un hábito alimenticio regular por parte del grupo estudiado. Al desglosar los factores se encontró en el consumo de azúcar una media de 3.22; la alimentación saludable presenta un 3.65; al preguntar sobre la realización de ejercicio físico la media fue de 3.17, en el contenido calórico el resultado fue de 3.35 , en 
el bienestar psicológico 3.56, en el tipo de alimentos 3.50, en el conocimiento y control de qué y cuánto comen con un valor de 3.01 y para el consumo de alcohol un 3.48 (figura 2).
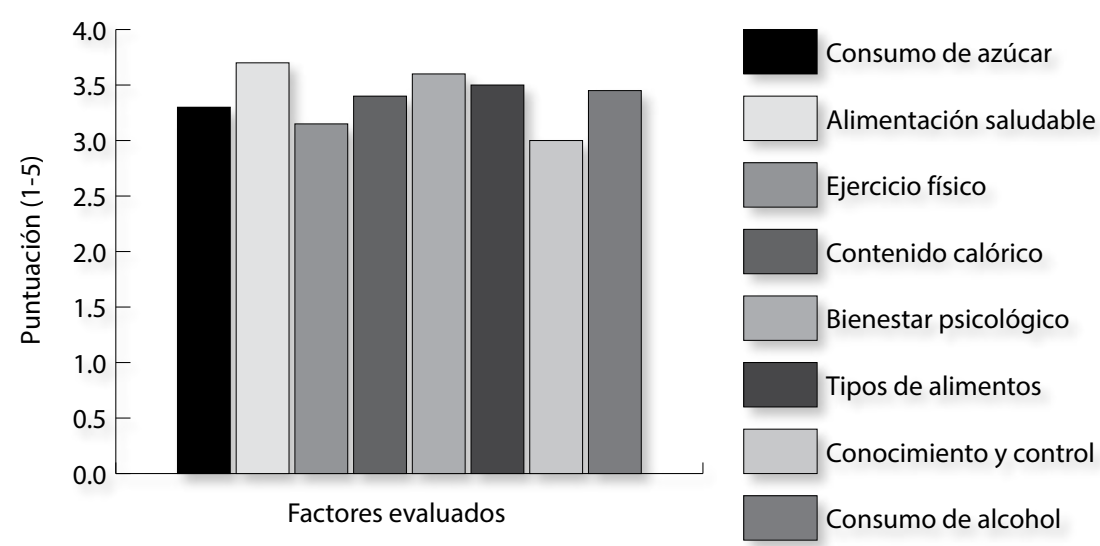

$$
\mathrm{n}=191 \text { alumnos }
$$

Figura 2. Distribución del promedio de los factores evaluados

En cuanto a la evaluación de los factores por semestre sobresale el grupo de octavo al obtener los valores más altos en alimentación saludable (4.0), ejercicio físico (3.44) y contenido calórico (3.88); el sexto semestre obtuvo el valor de la media en la ponderación alta en tipos de ali- mentos (3.68) y en el conocimiento y control (3.46); quinto semestre en consumo de azúcar (3.34) y bienestar psicológico (3.85), observando que el primero, segundo y tercer semestres mantienen promedios de 2 a 3 puntos en los ocho factores valorados (tabla 1).

\section{Tabla I}

\section{Media de los factores por semestre}

\begin{tabular}{|lllllllll|}
\hline Semestre & CAz & AS & EF & CC & BP & TA & CyC & CA \\
\hline $1^{\circ}$ & 3.13 & 3.37 & 3.13 & 2.98 & 3.37 & 3.48 & 2.94 & 3.48 \\
\hline $2^{\circ}$ & 3.11 & 3.51 & 3.24 & 3.33 & 3.66 & 3.34 & 2.86 & 3.72 \\
\hline $3^{\circ}$ & 3.25 & 3.65 & 2.81 & 3.33 & 3.56 & 3.65 & 2.94 & 3.51 \\
\hline $4^{\circ}$ & 3.26 & 3.67 & 2.95 & 3.13 & 3.84 & 3.38 & 2.6 & 3.45 \\
\hline $5^{\circ}$ & 3.34 & 3.84 & 3.28 & 3.44 & 3.85 & 3.63 & 3.12 & 3.40 \\
\hline $6^{\circ}$ & 3.22 & 3.65 & 3.34 & 3.53 & 3.41 & 3.68 & 3.4 & 3.37 \\
\hline $8^{\circ}$ & 3.33 & 4.08 & 3.44 & 3.88 & 3.15 & 3.41 & 3.33 & 3.23 \\
\hline
\end{tabular}

Caz = consumo de azúcar; $\mathrm{AS}=$ alimentación saludable; $\mathrm{EF}=$ ejercicio físico; $\mathrm{CC}=$ contenido calórico de la dieta; $\mathrm{BP}=$ bienestar psicológico; TA = tipos de alimentos que se consumen; $\mathrm{CyC}=$ conocimiento y control sobre lo que se come; $\mathrm{CA}=$ consumo de alcohol. 
Al cuestionar a los alumnos en cómo consideraban que era su alimentación se encontró que el $6 \%$ refirió tener una ali- mentación muy buena, el $46 \%$ buena, el $45 \%$ regular y sólo el $3 \%$ admitió que su alimentación es mala (figura 3).

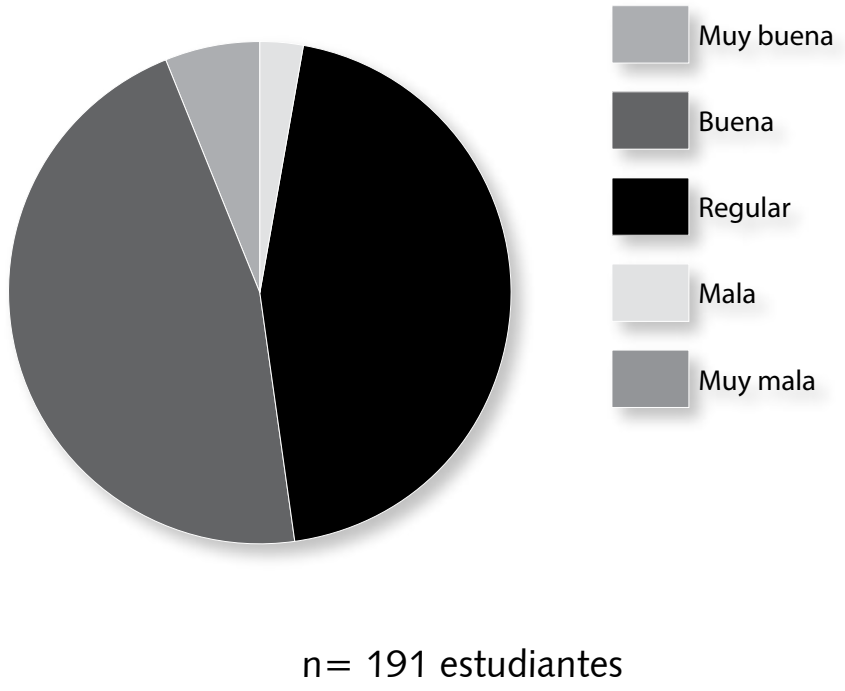

Figura 3: Percepción de hábitos alimenticios del grupo de estudio

\section{Discusión}

El cuestionario mediante el cual se evaluó a los alumnos consta de ocho factores diferentes que en conjunto forman los buenos hábitos alimenticios, dentro de los cuales se pudo observar que los alimentos que consumen los alumnos se ven limitados por factores económicos y por las restricciones del mercado ${ }^{5}$ en donde influye el factor de contenido calórico; esta posibilidad de disponer de diferentes opciones puede llegar a ser la que modifique los hábitos alimenticios, además de la falta de práctica de ejercicio físico y una inestabilidad psicológica por el estrés y la presión que genera el estudiar una carrera, además de una infinidad de factores que pueden llegar a modificar de manera inadecuada los hábitos alimenticios del alumno.

Se ha postulado que los hábitos alimentarios adquiridos durante la edad infantil y mantenidos durante la adolescencia persisten en la edad adulta. ${ }^{1}$ Esto puede verse modificado en los alumnos que estudian la carrera de Nutrición ya que, se podría pensar, que por tener conocimientos suficientes en cuanto a alimentación y estilo de vida saludable sus hábitos alimenticios podrían ser mejores; ${ }^{1}$ al presentar una media de 3,39 como resultado de hábitos alimenticios regulares en la población estudiada implica que no sólo se necesita tener conocimientos acerca de alimentación saludable sino que existen factores externos que pueden llegar a modificar los hábitos alimenticios de los alumnos; otro aspecto que cabe mencionar se refiere a si los estudiantes conservan un nivel adecuado de los conceptos en cuanto a alimentación otorgados en clase, lo cual puede ser importante al momento de elegir su dieta. ${ }^{6} \mathrm{El}$ que los alumnos de la carrera de nutrición lleven a cabo malos hábitos alimentarios puede deberse a sus gustos y preferencias sin importar el conocimiento adquirido. ${ }^{7}$ 
En los tres primeros semestres no se observaron valores altos en los promedios de ningún factor, lo que puede indicar que conforme el alumno va adquiriendo los conocimientos modifica sus hábitos alimenticios, lo cual se puede observar con los resultados de quinto y sexto semestre que alcanzan valores altos de medias en dos factores diferentes cada uno. De seguir así en octavo semestre sus hábitos alimenticios serían muy buenos, este semestre presenta valores promedio altos en cuanto a alimentación saludable, ejercicio físico y contenido calórico, lo que permite observar que a pesar de que son el grupo a formar parte de la vida profesional a corto plazo no llegan a medias altas en los ocho factores debido a diferentes situaciones como lo pueden ser los horarios diversos para realizar comidas, el tiempo disminuido para la preparación de alimentos, las prácticas hospitalarias por la mañana y clases por la tarde, la inapetencia por estrés, entre otros factores, ${ }^{5}$ se puede señalar que se torna difícil mantener buenos hábitos alimenticios a pesar de tener conocimientos de alimentación saludable.

\section{Conclusiones}

A pesar de tener conocimientos sobre una alimentación saludable, los estudiantes de la licenciatura de nutrición se ven influidos por diversos factores que afectan su forma de alimentarse. Es necesario enfatizar en el beneficio de adquirir mejores estrategias para preservar hábitos saludables.

\section{Bibliografía}

1. Montero $A B$, Úbeda NM, García AG. Evaluación de los hábitos alimentarios de una población de estudiantes universitarios en relación con sus conocimientos nutricionales. Nutr Hosp. 2006; 21(4):46673.

2. Espinoza LO, Rodríguez FR, Gálvez JC, MacMillan NK. Hábitos de alimentación y actividad física en estudiantes universitarios. Rev chil. nutr. 2011 Dic; 38(4): 458-465.

3. Gómez DJI, Salazar HN. Hábitos alimenticios en estudiantes Universitarios de ciencias de la salud de Minatitlán, Ver. [Tesis de licenciatura]. Minatitlan Veracruz: 2010. 1-39.

4. Castro RP. Elaboración y validación de un nuevo cuestionario de hábitos alimentarios para pacientes con sobrepeso y obesidad. Endocrinol Nutr. 2010; 57(4):130-139.

5. Rodríguez RF, Palma LX, Romo BA, Escobar BD, Aragú $\mathrm{GB}$, Espinoza $\mathrm{OL}$, et al. Hábitos alimentarios, actividad física y nivel socioeconómico en estudiantes universitarios de Chile. Nutr Hosp. 2013; 28(2):447455.

6. Vega MR, Ejeda Manzanera JM, Manjarrez González MT. Análisis de los conocimientos en alimentación de futuros sanitarios. Implicaciones pedagógicas. Teor. Educ. 22,1-2010::169-170.

7. Rodríguez $F R$, Palma $X L$, romo $A B$, escobar $D B$, Aragú $B G$. Hábitos alimentarios, actividad física y nivel socioeconómicos en estudiantes universitarios de Chile. Nutr Hosp, 2013;28(2):452. 